\title{
Working from home during the COVID-19 pandemic: surveys of the Society for Pediatric Radiology and the Society of Chiefs of Radiology at Children's Hospitals
}

\author{
Matthew C. Seghers ${ }^{1}$. Victor J. Seghers ${ }^{2,3} \cdot$ Andrew C. Sher ${ }^{2,3} \cdot$ Siddharth P. Jadhav $^{2,3} \cdot$ Lisa J. States ${ }^{4,5}$. \\ Andrew T. Trout ${ }^{6,7} \cdot$ Adina L. Alazraki $^{8,9} \cdot$ Marla B. K. Sammer $^{2,3}$ (D)
}

Received: 6 September 2021 / Revised: 19 December 2021 / Accepted: 23 January 2022 / Published online: 1 March 2022

(c) The Author(s), under exclusive licence to Springer-Verlag GmbH Germany, part of Springer Nature 2022

\begin{abstract}
Background Due to the COVID-19 pandemic, some pediatric radiologists have shifted to working from home; the long-term ramifications for pediatric radiologists and departments have not yet been defined.

Objective To characterize experiences of working from home associated with the COVID-19 pandemic and guide expectations after the pandemic is controlled, via separate surveys of Society for Pediatric Radiology (SPR) and Society of Chiefs of Radiology at Children's Hospitals (SCORCH) members.

Materials and methods Two separate surveys were conducted. In the first, SPR members were surveyed Jan. 11 through Feb. 8,2021 . The response rate was $17.0 \%$ ( 255 of 1,501). Survey questions included demographics, information on the ability to work from home and subjective experiences ranked on a scale of 0 to 10. The survey enabled segregation and comparison of responses between those with and without home PACS. In the second survey, SCORCH members were surveyed Dec. 8 , 2020, through Jan. 8, 2021. The response rate was 51.5\% (51/99). Survey questions included the logistics of working from home, technical specifications and the expectations on clinical duties performed from home. The Wilcoxon rank test was used to determine statistical significance of compared variables between respondents with and without home PACS in SPR members, and expectations between SPR and SCORCH members. Descriptive statistics summarized demographic questions and free text responses.

Results The majority of member respondents $(81.2 \%, 207 / 255)$ had a home PACS and most departments provided home PACS to faculty $(94.1 \%, 48 / 51)$. Overall, radiologists who could work from home were satisfied with their ability to work from home (mean rating: 8.3/10) and were significantly more satisfied than predicted by those without home PACS (5.9/10, $P<0.0001$ ). Respondents overwhelmingly indicated they were less able to teach trainees (mean rating: $2.7 / 10$ ) and had decreased emotional engagement (mean rating: 4.4/10), but had improved research productivity and cognitive ability for research when working from home (mean rating for both: 5.3/10). Regarding the expectations of the ability to work from home after no longer needing to address the pandemic, department chairs generally favored fewer rotations from home, with $97.9 \%$ (47/48) indicating working from home should be $60 \%$ or fewer assignments, compared with $84.1 \%(164 / 195)$ of individual radiologists $(P=0.071)$.

Conclusions Due to the COVID-19 pandemic, there has been a shift to working from home using PACS. Results of these SPR and SCORCH member surveys can help inform future decisions regarding pediatric radiologists working from home once the pandemic has been controlled.
\end{abstract}

Keywords COVID-19 · Emotional engagement · PACS · Pediatric radiology $\cdot$ Society for Pediatric Radiology $\cdot$ Society of Chiefs of Radiology at Children's Hospitals · Survey · Work from home

\section{Introduction}

Marla B. K. Sammer

msammer@gmail.com

Extended author information available on the last page of the article
The COVID-19 pandemic caused substantial disruptions to the United States health care industry and increased the use of telemedicine in multiple specialties [1-3]. Before this, the use of 
home Picture Archiving and Communication System (PACS) workstations was recommended by the American College of Radiology (ACR) for emergency scenarios though maintaining on-site radiologist coverage is preferred when possible [4]. However, a 2019 ACR member survey found that a majority of radiologists had performed teleradiology in the past decade, with many citing benefits of improving access for patients to after-hours care, and geographically dispersed and more specialized coverage by using teleradiology [5]. In early 2020, the Radiology journal editorial board completed an assessment of program preparedness for the pandemic, which recommended increasing off-site capabilities in the event of quarantines or surges in inpatient volume [6]. To address governmental "Stay Home, Work Safe" orders during the COVID-19 pandemic, many radiology departments moved rapidly to deploy home workstations and increase their capacity for off-site diagnostic responsibilities $[7,8]$.

Within pediatric radiology, the rationales for deploying home workstations during the pandemic included facilitating social distancing in the reading room, providing capacity for radiologists to isolate at home after exposure, and ensuring patient and staff safety [9]. The pandemic significantly accelerated existing work-at-home trends, forcing departments to quickly make major workforce decisions with incomplete information.

The purpose of this paper is to analyze the state of work-athome practices during the pandemic and the expectations for those practices going forward, via separate Society for Pediatric Radiology (SPR) and Society of Chiefs of Radiology at Children's Hospitals (SCORCH) member surveys, in order to guide future decision making under the assumption that workat-home practices will continue in some form even after the pandemic no longer needs to be addressed.

Both SPR and SCORCH member surveys targeted three aspects of the work-at-home trend. The first aimed to quantify the relative proportion of clinical rotations worked from home, including before the pandemic, during and projected after it is better controlled. Given separate surveys of leadership (SCORCH) and practitioners (SPR), attitudes and expectations were compared between groups. The second aim was to characterize the subjective effects of working from home felt by radiologists who have home PACS compared to radiologists who do not have PACS in the following domains: clinical workflows, education, communication, research and emotional engagement. Finally, a series of technical details were queried, with the aim to provide guidance for pediatric radiology departments.

\section{Materials and methods}

With separate SCORCH and SPR approval, surveys of the groups' members were created by seven of the authors (V.J.S., A.C.S., S.P.J., L.J.S., A.T.T., A.L.A., M.B.K.S., with 6 to 25 years of experience), who practice at four different children's hospitals. The 22-question SCORCH survey distributed via email to 99 members, ran from Dec. 8, 2020, through Jan. 8, 2021. The 23-question SPR survey (Online Supplementary Material 1), distributed via email to 1,501 members, ran from Jan. 11, 2021, through Feb. 8,2021 . One reminder email was sent before each survey closure date. Data were collected via Survey Monkey (Momentive Global Inc., San Mateo, CA) and responses were tabulated in Excel (Microsoft, Redmond, WA). This study was exempt from institutional review board approval.

Three questions from the SPR survey were demographic, requesting the respondent's type of SPR membership category, whether the respondent had a home PACS and the size of their radiology group. Geographical location was not queried or used to filter recipients, only member type. While most SPR survey recipients reside within North America, some do not. Four questions from the SCORCH survey were demographic, requesting the name of the respondent's institution, the size of their pediatric radiology group, and whether they provided home workstations to faculty and/or trainees to enable working from home. Of the $99 \mathrm{SCORCH}$ recipients, 7 were from Canadian institutions $(7.1 \%, 7 / 99$ total recipients outside the United States).

Two questions appeared on both surveys, detailing the percentage of clinical rotations worked from home during and predicted after the COVID-19 pandemic is controlled, enabling comparison of responses between pediatric radiology practitioners and departmental leadership.

The remainder of the SPR survey questions were opinion-based. For these questions, respondents were asked to rank their experience on a 0-10 numeric Likert scale comparing work at home versus in-hospital, with a score of 5 representing the same experience at home versus in-hospital, a score of 0 representing a maximum diminishment in the experience of work from home versus in-hospital, and a score of 10 representing a maximum improvement in the experience of work from home versus in-hospital. For respondents without a home PACS workstation and unable to work at home, survey questions were modified asking for their impression of what they believe the work-fromhome experience might be like. This approach allowed for comparisons between the two groups, providing a means to examine individuals' beliefs about what the experience of home PACS might be like, compared to the actual experience of using a home PACS. These questions examined levels of satisfaction, feelings of professional accomplishment, challenges and benefits from working from home. Additional questions examined issues of clinical productivity, diagnostic accuracy, communication, ability to teach trainees, and research productivity when working 
from home to reflect many academic radiologists' clinical, educational and research missions.

In the SCORCH survey, nine questions discussed technical aspects of their department's home PACS setup. Five questions involved operational and communication workflows while working from home.

Each survey concluded with a free-text question. Free text responses were categorized as positive, mixed or negative by three authors (V.J.S., A.C.S., S.P.J.). Free-text responses were also categorized according to the topics covered. For the SPR survey, these included clinical workflows, teaching of trainees, convenience and flexibility, and concerns regarding disconnects between radiology departments and other in-hospital teams. For the SCORCH survey, these included clinical workflows (such as issues of work-life balance, commuting times, leadership, participation in meetings, communication with ordering providers, technical aspects of home PACS), teaching of trainees or research. Differences in categorization between the reviewing authors were resolved by consensus.

For statistical evaluation, individual responses were deidentified and coded based on queried variables. Wilcoxon rank sum test was used to determine the statistical significance of compared variables. Descriptive statistics were used to summarize demographic questions and free-text responses.

\section{Results}

Of the 1,501 SPR members surveyed, 255 recipients responded ( $17.0 \%$ response rate). There were 216 complete (84.7\%) and 39 partial (15.3\%) responses. Ninety-seven percent (247/255) of the respondents were active SPR members and $3 \%$ were trainees $(8 / 255)$. The majority of respondents (207/255, 81.2\%) had a home PACS station. Most respondents $(164 / 253,64.8 \%)$ practiced in groups of 20 or fewer radiologists (Table 1). Most (97.3\% [248/255]) of the SPR member respondents resided in the United States, with $2 \%(5 / 255)$ in Canada and $0.8 \%$ (2/255) outside of North
America. In the SCORCH survey, all respondents were from U.S. institutions.

There were 51 SCORCH respondents $(51.5 \%$ response rate). Most respondents $(80.4 \%, 41 / 51$ respondents) were in groups of 20 or fewer radiologists (Table 1). Most respondents $(94.1 \%, 48 / 51)$ indicated home PACS are provided to faculty in their department. For the 3 institutions that did not provide home PACS, it was planned to be deployed in $2(66.7 \%)$ over the next 1-2 years. We found no significant difference in providing home PACS based on group size $(P=0.38)$, with $97.4 \%$ (37/38) of smaller group $(<21$ radiologists) respondents providing home PACS vs. $90.0 \%(9 / 10)$ of larger groups (21 or more radiologists).

\section{Work-at-home trends}

Before the pandemic (defined as before March 2020), SCORCH respondents indicated that faculty worked very few clinical rotations from home, with $20 \%$ or fewer rotations worked from home in $93.8 \%$ of departments before the pandemic. During the pandemic (defined as March 2020-December 2020 in the surveys), respondents indicated that work from home increased. Specifically, before the pandemic 50.0\% (24/48) reported no clinical work rotations were performed from home, which decreased to only $4.2 \%$ (2/48) departments during the pandemic (Table 2). Before the pandemic, the departments that allowed workfrom-home assignments did so for only a minority of overall clinical effort, with none reporting $>41 \%$ of rotations from home versus in-hospital. In contrast, during the pandemic, the amount of at-home rotations increased dramatically, with $47.9 \%$ (21/48) of SCORCH respondents reporting that staff worked $41-99 \%$ of clinical rotations from home. The majority of respondents $(52.1 \%, 25 / 48)$, however, reported that only $40 \%$ or fewer rotations were staffed from home during the pandemic. This is in concordance with reporting from a majority of individual radiologists via the SPR survey $(55.2 \%, 107 / 194)$ who also indicated $40 \%$ or fewer rotations were staffed from home.
Table 1 Society for Pediatric Radiology (SPR) and Society of Chiefs of Radiology at Children's Hospitals (SCORCH) respondents' group sizes

\begin{tabular}{lllll}
\hline $\begin{array}{l}\text { Group size (number } \\
\text { radiologists in group) }\end{array}$ & $\begin{array}{l}\text { Number of SPR } \\
\text { respondents }\end{array}$ & $\begin{array}{l}\text { Percent of SPR } \\
\text { respondents }\end{array}$ & $\begin{array}{l}\text { Number of } \\
\text { SCORCH respond- } \\
\text { ents }\end{array}$ & $\begin{array}{l}\text { Percent of } \\
\text { SCORCH } \\
\text { respondents }\end{array}$ \\
\hline$<6$ & 61 & $24.1 \%$ & 13 & $25.5 \%$ \\
$6-10$ & 60 & $23.7 \%$ & 17 & $33.3 \%$ \\
$11-20$ & 43 & $17.0 \%$ & 11 & $21.6 \%$ \\
$21-30$ & 47 & $18.6 \%$ & 6 & $11.8 \%$ \\
$31-40$ & 24 & $9.5 \%$ & 2 & $3.9 \%$ \\
$>40$ & 18 & $7.1 \%$ & 2 & $3.9 \%$ \\
\hline
\end{tabular}

Distribution was similar in both survey respondent groups 
Table 2 Summary of rotations worked from home before, during and projected after the pandemic

\begin{tabular}{lllllll}
\hline & $\begin{array}{l}\text { Before pandemic } \\
\begin{array}{l}\text { Percent of assign- } \\
\text { ments at home }\end{array}\end{array}$ & $\begin{array}{l}\text { Furing the pandemic } \\
\text { Fy department* }\end{array}$ & $\begin{array}{l}\text { Frequency and percent } \\
\text { by department }\end{array}$ & $\begin{array}{l}\text { Frequency and percent } \\
\text { by radiologist }\end{array}$ & $\begin{array}{l}\text { Frequency and percent } \\
\text { by department }\end{array}$ & $\begin{array}{l}\text { Frequency and } \\
\text { percent by radi- } \\
\text { ologist }\end{array}$ \\
\hline None & $50.0 \%(24 / 48)$ & $4.2 \%(2 / 48)$ & $4.6 \%(9 / 194)$ & & $8.3 \%(4 / 48)$ & $5.6 \%(11 / 195)$ \\
$1-20 \%$ & $43.8 \%(21 / 48)$ & $22.9 \%(11 / 48)$ & $27.3 \%(53 / 194)$ & & $41.7 \%(20 / 48)$ & $32.8 \%(64 / 195)$ \\
$21-40 \%$ & $6.3 \%(3 / 48)$ & $25.0 \%(12 / 48)$ & $23.2 \%(45 / 194)$ & & $35.4 \%(17 / 48)$ & $27.7 \%(54 / 195)$ \\
$41-60 \%$ & $0 \%$ & $16.7 \%(8 / 48)$ & $15.5 \%(30 / 194)$ & & $12.5 \%(6 / 48)$ & $18.0 \%(35 / 195)$ \\
$61-80 \%$ & $0 \%$ & $25.0 \%(12 / 48)$ & $13.4 \%(26 / 194)$ & $2.1 \%(1 / 48)$ & $6.2 \%(12 / 195)$ \\
$81-99 \%$ & $0 \%$ & $6.3 \%(3 / 48)$ & $6.7 \%(13 / 194)$ & & $0 \%$ & $4.1 \%(8 / 195)$ \\
$100 \%$ & $0 \%$ & $0 \%$ & $9.3 \%(18 / 194)$ & & $0 \%$ & $5.6 \%(11 / 195)$ \\
\hline
\end{tabular}

Results are provided for both Society of Chiefs of Radiology at Children's Hospitals (SCORCH) (department) respondents and individual radiologists who have the ability to work from home using PACS* in the Society for Pediatric Radiology (SPR) survey. General trends show an increase in the amount of at-home clinical work rotations during the pandemic relative to before from both pediatric radiology department chairs and individual radiologists, a trend that is expected to continue after the pandemic is better controlled. However, departmental chairs generally favored fewer rotations from home once the pandemic is better controlled relative to individual radiologists

*Before pandemic was queried of departments only, not of individual radiologists in the SPR survey

After the pandemic is controlled, 91.7\% (44/48) of SCORCH respondents predicted that some rotations could be performed from home, though the majority $(77.1 \%, 37 / 48)$ predicted this would only affect between $1 \%$ and $40 \%$ of rotations (Table 2). While the proportion of respondents supporting 1-20\% of at-home rotations did not substantially change from before the pandemic $(43.8 \%, 21 / 48$ before the pandemic vs. $41.7 \%, 20 / 48$ predicted after the pandemic), there was a substantial shift away from working entirely in the hospital after the pandemic is better controlled. For example, before the pandemic, 50.0\% (24/48) of responding departmental leaders reported "none" to reasonable proportion of rotations to work from home vs. $8.3 \%$ (4/48) projected after the pandemic is controlled.

Regarding working from home post-pandemic, SCORCH respondents generally favored fewer rotations from home, with $97.9 \%$ (47/48) indicating working from home should be $60 \%$ or fewer assignments, compared with $84.1 \%(164 / 195)$ of individual radiologists (Table 2), but this difference was not statistically significant $(P=0.071)$. Conversely, $15.9 \%(31 / 195)$ of individual radiologists reported it would be reasonable to work $61 \%$ or greater rotations at home after the pandemic, compared to $2.1 \%$ (1/48) of department chairs. Finally, no departments reported working $100 \%$ of rotations from home was reasonable, compared to $5.6 \%$ (11/195) of individual radiologists.

\section{Effects of working from home as indicated by SPR respondents}

Overall, respondents who worked from home using a PACS workstation were satisfied with their ability to work from home (mean rating: 8.3 out of 10). The benefits of working from home identified by respondents are outlined in Table 3. For respondents who have home PACS, the lack of a commute, the flexibility in work schedule and improved efficiency in participating remotely in meetings were identified by the majority as benefits.

Challenges of working from home are outlined in Table 4. Changes in social interactions with colleagues, differences in communications, and difficulty with social and emotional engagement were identified as challenges by the majority of respondents who have home PACS.

Results of subjective differences in working from home compared to in the hospital are summarized in Table 5. Notably, respondents with home PACS reported slightly increased clinical productivity from home compared to in the hospital (mean rating: 5.8). They also reported similar to slightly improved diagnostic accuracy when working from home (mean rating: 5.2). However, the ability to communicate with referring providers was scored as similar to slightly more difficult when working from home compared to in the hospital (mean rating: 4.8).

\section{Teaching and research}

As noted in Table 5, SPR respondents who have home PACS overwhelmingly indicated that they were less able to teach trainees when working from home (mean 
Table 3 Reported benefits of working from home by Society for Pediatric Radiology respondents with ( $n=194$ respondents to question) and without home PACS systems ( $n=43$ respondents to question)

\begin{tabular}{|c|c|c|c|}
\hline Answer choices to BENEFITS of working from home & $\begin{array}{l}\text { Respondents } \\
\text { with home } \\
\text { PACS } \\
\text { \# and \% }\end{array}$ & $\begin{array}{l}\text { Respondents without } \\
\text { home PACS, \# and \% }\end{array}$ & $\begin{array}{l}\text { Comparison } \\
(P \text {-value })\end{array}$ \\
\hline Lack of commute & $179(92.3 \%)$ & $38(88.4 \%)$ & 0.37 \\
\hline Flexibility in work schedule & $142(73.2 \%)$ & $32(74.4 \%)$ & 1.00 \\
\hline $\begin{array}{l}\text { Improved efficiency when participating remotely in meetings/multidisciplinary confer- } \\
\text { ences }\end{array}$ & $108(55.7 \%)$ & $16(37.2 \%)^{*}$ & 0.04 \\
\hline Decreased distractions compared with working in the hospital/clinic & $95(49.0 \%)$ & $11(25.6 \%)^{*}$ & 0.006 \\
\hline $\begin{array}{l}\text { Flexibility when you or your child are mildly ill (e.g., can work from home without } \\
\text { requiring a sick day and not expose coworkers) }\end{array}$ & $84(43.3 \%)$ & $21(48.8 \%)^{*}$ & 0.61 \\
\hline $\begin{array}{l}\text { Childcare (not needing childcare or being able to interact with your children during the } \\
\text { workday) }\end{array}$ & $64(33.0 \%)$ & $4(9.3 \%)^{*}$ & 0.001 \\
\hline Physical workspace & $43(22.2 \%)$ & $8(18.6 \%)^{*}$ & 0.69 \\
\hline $\begin{array}{l}\text { No change in rotations (e.g., structure of in-hospital rotations/responsibilities/time frame } \\
\text { did NOT change despite now working from home) }\end{array}$ & $12(6.2 \%)$ & $1(2.3 \%)$ & 0.47 \\
\hline $\begin{array}{l}\text { Differences in communications (coworkers, technologists, ordering providers, nursing } \\
\text { staff, etc.) }\end{array}$ & $11(5.7 \%)$ & 0 & 0.22 \\
\hline $\begin{array}{l}\text { Change in social interactions with colleagues (both within and outside Department of } \\
\text { Radiology) }\end{array}$ & $7(3.6 \%)$ & 0 & 0.36 \\
\hline Social or emotional engagement with hospital/coworkers & $7(3.6 \%)$ & 0 & 0.36 \\
\hline
\end{tabular}

The top three benefits from each cohort are in bold

*Variables with different ranking from the group who were not able to work from home, compared to those who do work from home

Table 4 Reported challenges of working from home by Society for Pediatric Radiology respondents with ( $n=191$ respondents to question) and without home PACS systems ( $n=44$ respondents to question)

\begin{tabular}{|c|c|c|c|}
\hline Answer choices to CHALLENGES when working from home & $\begin{array}{l}\text { Respondents with } \\
\text { home PACS } \\
\text { \# and } \%\end{array}$ & $\begin{array}{l}\text { Respondents without } \\
\text { home PACS } \\
\text { \# and \% }\end{array}$ & $\begin{array}{l}\text { Comparison } \\
(P \text {-value })\end{array}$ \\
\hline $\begin{array}{l}\text { Change in social interactions with colleagues } \\
\text { (both within and outside Department of Radiology) }\end{array}$ & $135(70.1 \%)$ & $37(84.1 \%)$ & 0.09 \\
\hline $\begin{array}{l}\text { Differences in communications } \\
\text { (coworkers, technologists, ordering providers, nursing staff, etc.) }\end{array}$ & $110(57.6 \%)$ & $36(81.8 \%)$ & 0.003 \\
\hline Social or emotional engagement with hospital/coworkers & $102(53.4 \%)$ & $27(61.4 \%)$ & $\mathbf{0 . 4 0}$ \\
\hline Decreased boundaries between work responsibilities and home/personal life & $74(38.7 \%)$ & $21(47.7 \%)$ & 0.31 \\
\hline Internet connectivity/power outages & $50(26.2 \%)$ & $20(45.5 \%)$ & 0.02 \\
\hline Computing hardware/software difficulties & $38(19.9 \%)$ & $14(31.8 \%)$ & 0.11 \\
\hline Childcare (both availability of child care or simply having children at home) & $33(17.3 \%)$ & $10(22.7 \%)$ & 0.39 \\
\hline Physical workspace & $29(15.2 \%)$ & $10(22.7 \%)$ & 0.26 \\
\hline Too many distractions at home & $28(14.7 \%)$ & $17(38.6 \%)^{*}$ & 0.001 \\
\hline Lack of usual reference materials at home & $25(13.1 \%)$ & $4(9.1 \%)$ & 0.61 \\
\hline Pressure to still work from home despite being mildly ill & $15(7.9 \%)$ & $3(6.8 \%)$ & 1.00 \\
\hline $\begin{array}{l}\text { No change in rotations } \\
\text { (e.g., structure of in-hospital rotations/responsibilities/time frame did NOT } \\
\text { change despite now working from home) }\end{array}$ & $15(7.9 \%)$ & $1(2.3 \%)$ & 0.32 \\
\hline
\end{tabular}

The top three challenges from each cohort are in bold

*Variable with different ranking from the group who were not able to work from home, compared to those who do work from home 
Table 5 Mean rankings on the subjective differences when working from home compared to in the hospital or clinic for Society for Pediatric Radiology respondents

\begin{tabular}{|c|c|c|c|}
\hline Question* & $\begin{array}{l}\text { Home PACS } \\
\text { (mean, SD) }\end{array}$ & $\begin{array}{l}\text { No home PACS* } \\
\text { (mean, SD) }\end{array}$ & $\begin{array}{l}\text { Comparison } \\
(P \text {-value })\end{array}$ \\
\hline How SATISFIED is respondent with ability to work from home & $8.3(2.2)$ & $5.9(3.1)$ & $<0.0001$ \\
\hline Difference in CLINICAL PRODUCTIVITY when working from home & $5.8(2.1)$ & $5.6(1.9)$ & 0.58 \\
\hline $\begin{array}{l}\text { Difference in COGNITIVE ABILITY for RESEARCH } \\
\text { when working from home }\end{array}$ & $5.3(2.2)$ & $4.5(1.7)$ & 0.08 \\
\hline $\begin{array}{l}\text { Difference in RESEARCH PRODUCTIVITY } \\
\text { when working from home }\end{array}$ & $5.3(2.3)$ & $4.7(1.9)$ & 0.09 \\
\hline Difference in DIAGNOSTIC ACCURACY when working from home & $5.2(1.3)$ & $5.1(1.3)$ & 0.26 \\
\hline $\begin{array}{l}\text { Difference in ability to MEANINGFULLY participate in departmental and interdepart- } \\
\text { mental meetings from home }\end{array}$ & $5.2(2.2)$ & $4.1(2.0)$ & 0.005 \\
\hline Difference in sense of PROFESSIONAL ACCOMPLISHMENT when working from home & $5.0(2.3)$ & $3.5(2.2)$ & $<0.0001$ \\
\hline $\begin{array}{l}\text { Difference in ability to COMMUNICATE WITH REFERRING PROVIDERS when work- } \\
\text { ing from home }\end{array}$ & $4.8(1.7)$ & $3.8(1.8)$ & 0.0007 \\
\hline $\begin{array}{l}\text { Difference in ability to COMMUNICATE with RESEARCH team when working from } \\
\text { home }\end{array}$ & $4.6(1.5)$ & $4.0(1.7)$ & 0.02 \\
\hline Increased need for WELLNESS programs in hospital & $4.6(1.9)$ & $5.3(2.1)$ & 0.07 \\
\hline Difference in level of EMOTIONAL ENGAGEMENT when working from home & $4.4(1.8)$ & $3.2(2.0)$ & 0.002 \\
\hline Difference in ability to TEACH learners when working from home & $2.7(1.9)$ & $2.2(1.8)$ & 0.14 \\
\hline
\end{tabular}

Responses were ranked on a scale of 0 to 10 , with 0 a strongly decreased experience when working from home compared to in-hospital, 5 defined as no difference, and 10 defined as a strongly improved experience from home. Experiences that are better from home for the group who are able to work from home are listed in rank order. Statistically significant differences between anticipated experience from those without home PACS ( $n=48$ respondents) vs. actual with home PACS ( $n=207$ respondents) are bold

*For those without home PACS, the questions posed to the work-from-home group were modified to indicate that answer was based on how participants anticipated they would feel about working from home, if given the opportunity

Table 6 Relationship with trainees

\begin{tabular}{|c|c|c|c|}
\hline $\begin{array}{l}\text { Answer choices exploring how working from home has affected ability to } \\
\text { interact with trainees }\end{array}$ & $\begin{array}{l}\# \text { and } \% \text { of respond- } \\
\text { ents with home } \\
\text { PACS }\end{array}$ & $\begin{array}{l}\# \text { and } \% \text { of respondents } \\
\text { without home PACS }\end{array}$ & $\begin{array}{l}\text { Comparison } \\
\text { ( } P \text {-value })\end{array}$ \\
\hline More difficult to interact with trainees & $125(73.5 \%)$ & $36(90.0 \%)$ & 0.04 \\
\hline Easier to interact with trainees & $7(4.1 \%)$ & $1(2.5 \%)$ & 1.00 \\
\hline More difficult to staff cases from home without ability to illustrate findings & $91(53.5 \%)$ & $26(65.0 \%)$ & 0.22 \\
\hline Easier to staff cases from home using screen sharing & $29(17.1 \%)$ & $3(7.5 \%)$ & 0.15 \\
\hline More difficult to participate in teaching conferences from home & $53(31.2 \%)$ & $22(55.0 \%)$ & 0.006 \\
\hline Easier to participate in teaching conferences from home & $69(40.6 \%)$ & $6(15.0 \%)$ & 0.003 \\
\hline More difficult to provide didactic teaching from home & $65(38.2 \%)$ & $24(60.0 \%)$ & 0.02 \\
\hline Easier to provide didactic teaching from home & $48(28.2 \%)$ & $2(5.0 \%)$ & 0.002 \\
\hline
\end{tabular}

Nearly all queried interactions were believed to be more difficult when working from home by a majority of the Society for Pediatric Radiology respondents who use home PACS $(n=170)$ or anticipated by those without home PACS $(n=40)$. Answer choices receiving a majority response are in bold

rating: 2.7). Table 6 further details changes in working with trainees by querying whether certain activities were more difficult or easier when working from home. Notably, respondents working from home generally found it more difficult to interact with trainees $(73.5 \%, 125 / 170$ more difficult vs. $4.1 \%, 7 / 170$ easier), more difficult to staff cases from home with a trainee $(53.5 \%, 91 / 170$ more difficult vs. $17.1 \%, 29 / 170$ easier) and more difficult to provide didactic teaching $(38.2 \%, 65 / 170$ more difficult vs. $28.2 \%$, $48 / 170$ easier). The only improved interaction reported was the ability to participate in teaching conferences from home $(40.6 \%, 69 / 170$ easier vs. $31.2 \%, 53 / 170$ more difficult). Comparatively, SPR respondents who did not have home PACS universally expected the queried educational interactions to be more difficult from home, rather than easier. 
Respondents who have home PACS indicated their research productivity and cognitive ability for research were slightly improved from home compared to in the hospital (mean rating for both: 5.3, Table 5). However, they also reported it was more difficult to communicate with the research team when working from home (mean rating: 4.6).

\section{Emotional engagement}

Respondents who have home PACS indicated slightly improved ability to participate meaningfully in departmental meetings, teaching conferences, and visiting professorship lectures when working from home (mean rating: 5.2, Table 5). However, they reported less emotional engagement with their institution when working from home, compared to in the hospital (mean rating: 4.4). They also reported that there was less perceived need for wellness programs when working in the hospital compared to home (mean rating: 4.6).

Table 7 outlines the methods departments used to improve emotional engagement for individuals working from home and their perceived usefulness by responding radiologists. The only method identified by more than half the respondents who have home PACS as improving emotional engagement was the creation of hybrid schedules enabling some clinical rotations to be performed in the hospital, and not work solely from home. Other methods of increased connection through more intradepartmental meetings, the use of video in addition to audio at meetings, and increased social virtual events were found to be helpful by about a third of the respondents who have home PACS.

\section{Expectations of those without home PACS compared to those with home PACS}

As a group, radiologists who did not have home PACS anticipated fewer benefits of working from home compared to those who could work from home (Table 3). Conversely, they also anticipated more challenges (Table 4).
In addition to studying subjective differences when working from home compared to in the hospital by SPR respondents, questions were modified for those without home PACS to compare actual versus anticipated experiences (Table 5). Respondents with home PACS were significantly more satisfied with working from home relative to predictions by those without home PACS (mean rating: 8.3 vs. $5.9, P<0.0001)$. Respondents with home PACS reported a significantly greater sense of professional accomplishment than predicted by those without home PACS (mean: 5.0 vs. 3.5, P<0.0001). Those with home PACS also reported a significantly greater ability to communicate with referring providers from home than projections by those without home PACS (mean: 4.8 vs. 3.8, $P=0.0007)$. Similarly, respondents with home PACS reported a greater ability to communicate with the research team than anticipated by those without home PACS (mean: 4.6 vs. $4.0, P=0.02$ ). However, with mean ratings $<5.0$ for both communications questions, there was agreement in that respondents with and without home PACS experienced or expected to experience a decrease in the ability to communicate with both referring providers and the research team from a baseline of working in the hospital or clinic. Those with actual experience working from home found the communication issues less challenging relative to what those without home PACS anticipated. Those with home PACS additionally reported improved ability to meaningfully participate in departmental meetings, conferences and visiting professorship lectures from home, compared to what those without home PACS anticipated (mean: 5.2 vs. 4.1, $P=0.005$ ). Finally, respondents with home PACS reported greater emotional engagement with their institution than those without home PACS predicted (mean: 4.4 vs. $3.2, P=0.002$ ), although as with the communication questions, this represented a decrease in emotional engagement relative to what was felt when working in hospital.

Table 7 Methods endorsed to improve emotional engagement compared for Society for Pediatric Radiology respondents with ( $n=137)$ and without ( $n=39$ respondents) home PACS systems

\begin{tabular}{|c|c|c|c|}
\hline $\begin{array}{l}\text { Answer choices exploring methods to improve emotional engagement with the workplace } \\
\text { when working from home }\end{array}$ & $\begin{array}{l}\text { Respondents } \\
\text { with home } \\
\text { PACS } \\
\text { \# and \% }\end{array}$ & $\begin{array}{l}\text { Respondents } \\
\text { without home } \\
\text { PACS } \\
\text { \# and \% }\end{array}$ & $\begin{array}{l}\text { Comparison } \\
(P \text {-value })\end{array}$ \\
\hline $\begin{array}{l}\text { Creation of hybrid schedules to ensure that people still work some shifts in the hospital and } \\
\text { do not solely work from home }\end{array}$ & $99(72.3 \%)$ & $35(89.7 \%)$ & 0.03 \\
\hline Increased connection through more frequent faculty/division meetings & $45(32.9 \%)$ & $7(18.0 \%)$ & 0.08 \\
\hline Expectation that video and not just audio is utilized during meetings & $45(32.9 \%)$ & $21(53.9 \%)$ & 0.02 \\
\hline $\begin{array}{l}\text { Increased virtual events not solely focused on clinical/educational work (e.g., virtual happy } \\
\text { hour, virtual coffee break, group exercise, mindfulness events) }\end{array}$ & $43(31.4 \%)$ & $14(35.9 \%)$ & 0.70 \\
\hline
\end{tabular}




\section{Free-text question responses}

The free-text question "Anything you would like to convey to the pediatric radiology community about working from home?" was offered to all respondents. Excluding responses such as "No," "N/A" and "Not really," there were 112 and 20 responses from those with and without home PACS, respectively. For those with home PACS, responses were mostly positive $(63.4 \%, n=71)$, and less frequently negative $(25.0 \%$, $n=28)$ or mixed $(11.6 \%, n=13)$. For those without home PACS, responses were mostly positive $(55 \%, n=11)$ and less frequently negative $(45.0 \%, n=9)$ with no mixed responses.

For those with a home PACS, 85.7\% ( $n=96)$ of responses concerned workflow, $8.0 \%(n=9)$ involved teaching of trainees and $6.3 \%(n=7)$ encompassed a variety of themes. Almost all positive comments reflected improvements in clinical workflows, touching on issues of flexibility, better work-life balance, decreased feelings of burnout, and increased productivity and efficiency. One positive comment linked improved productivity in manuscript creation to time savings from decreased commutes to work. Of note, free-text responses regarding teaching of trainees were uniformly negative, focusing on challenges with communication and engagement. Negative comments regarding clinical work focused on technical issues (slow connectivity to PACS, difficulty managing electronic communication, slow responsiveness of informatics support personnel), perceived inequality of access to at-home work rotations, poor communication with ordering providers and patients, and increased feelings of detachment from the radiology department. A sample of free-text responses to the SPR survey sorted as positive, negative or mixed are listed in Table 8 .

For those without home PACS, $95.0 \%(n=19)$ of the responses concerned workflow. While the majority of comments from this group were positive, emphasizing desired flexibility for both in-hospital and at-home work rotations and potential improvement in work-life balance, negative responses tended to focus on issues of leadership regarding being at home while other members of the team were in-hospital, potential unequal access to at-home rotations for those with predominantly ultrasound and fluoroscopic responsibilities, and commoditization of radiology services due to a perceived lack of engagement with hospital personnel and consulting staff.

There were 26 responses to the free-text question on the SCORCH survey: "Anything you would like to convey to the SCORCH community about working from home and home PACS?" Of the responses, 11 (42.3\%) were mixed positive and negative, $8(30.8 \%)$ positive and $7(26.9 \%)$ negative. Among the negative responses, 6 (29\%) mentioned concern that working clinically from home eroded relationships between radiology departments and other staff including technologists, referring providers, consulting teams and nursing departments. Specifically, a common theme was that by operating too much from home, institutions may view radiology departments as similar to teleradiology services and jeopardize relationships. Five (24\%) of the responses mentioned teaching trainees and were uniformly negative, focusing on the difficulty that programs had remotely teaching trainees from home. Five (24\%) mentioned clinical workflows and were positive, indicating that home PACS systems could be used for emergency staffing issues, weekends or call shifts. Finally, 4 of 26 (19\%) respondents mentioned improved flexibility and convenience with child or elder care as positive aspects of home PACS systems. A sample of free-text responses to the SCORCH survey sorted as positive, negative or mixed are listed in Table 9.

Table 8 Sample of free-text responses from Society for Pediatric Radiology survey respondents who have home PACS ( $n=112$ ) to the question "Anything you would like to convey to the pediatric radiology community about working from home?"

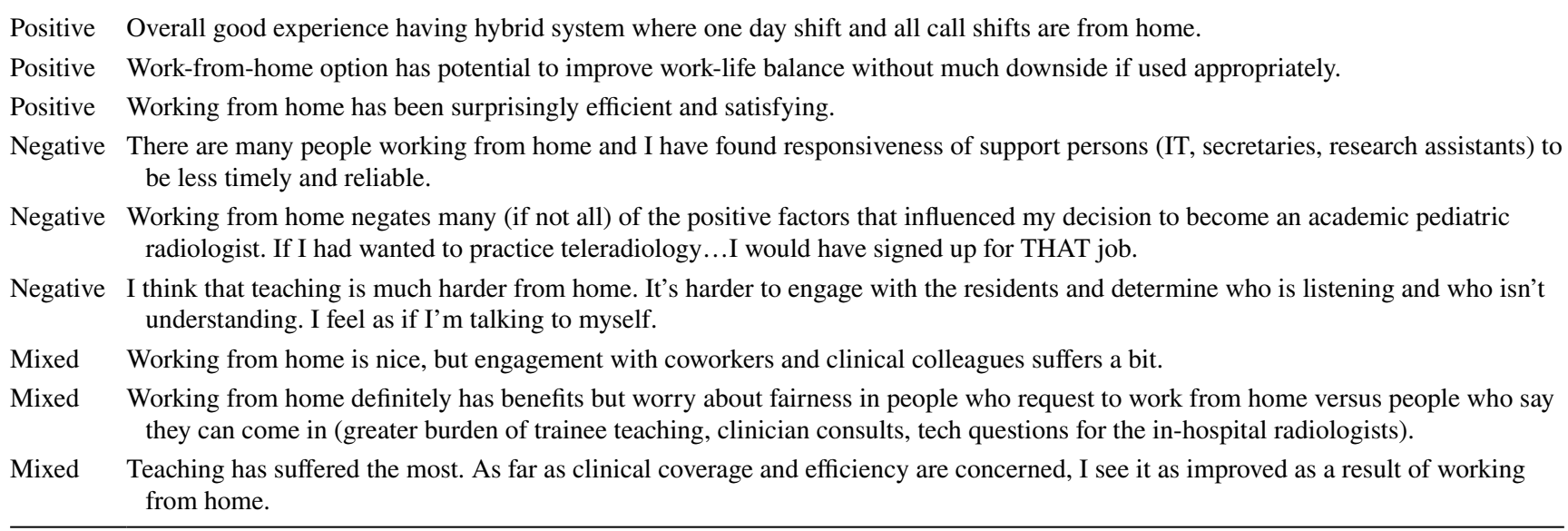

Responses were sorted as positive, negative or mixed. Comments lightly edited for clarity and grammar 
Table 9 Sample of free-text responses from Society of Chiefs of Radiology at Children's Hospitals survey respondents ( $n=26)$ to the question "Anything you would like to convey to the pediatric radiology community about working from home?"

Positive Essential part of practice. Full office PACS should be at home. Significantly improves patient care and work/life balance.

Positive Great for the evening shift, call, weekends, unexpected child care challenges, working while quarantined.

Positive Gained reading efficiency. As surgeons and oncologists seem to be only clinical teams that come down to review cases in real-time, those relationships are slightly lessened, although we always have someone in house for them to consult (it might not be the original reader, though). Pediatric medical teams seem to really like Zoom rounds more than in person, go figure?

Negative I strongly feel we should limit working from home during regular hours. Everything we are trying to do these days revolves around engaging MORE with referring providers and INCREASING our presence. Remote reading puts us at serious risk of being replaced or commoditized.

Negative Big issue concerning education - pediatric residents/medical students/fellows with attendings at home. The spontaneous side-by-side teaching has decreased.

Negative Home system is a bit slower and Powerscribe sometimes get stuck, which is supposedly related to constraints of the VPN.

Mixed I am grateful for the technology but have concerns about erosion of radiologist on site presence that could diminish our visibility and value-added. Also, I don't think many faculty are as engaged working from home, which is always a challenge anyway.

Mixed As a radiologist I like it- as the [program director] for our residency I think it is terrible and I worry about the amount of feedback our trainees get about small nuances in reports, etc.

Mixed We love working from home, especially people with young children and some of us with aged parents. It is more efficient as the interruptions are less. Disadvantages: limited interaction with clinicians, have not been able to change the culture, limited interaction with technologists, most important, one on one teaching, interaction and mentoring of trainees is severely impaired despite Zoom et al. For that one reason, and resentment from clinicians, we have gone back to mostly in-person reading at hospital when safe, and now with all of us having received our 1 st vaccine dose.

Responses were sorted as positive, negative or mixed. Comments lightly edited for clarity and grammar

\section{Technical specifications and operational logistics as reported by department chairs}

Funding for home workstations was most commonly from radiology department funds $(48.9 \%, 23 / 47)$ or hospital funds $(44.7 \%, 21 / 47)$. In a minority, they were funded via professional expense account $(4.3 \%, 2 / 47)$ or self-pay $(2.1 \%$, $1 / 47)$. The majority of SCORCH respondents $(54.2 \%, 26 / 48)$ deployed the same monitors (number and type) for radiologists to use at home that were used in the hospital. The types of reading workstations varied by program, though the majority of programs $(95.1 \%, 39 / 41)$ provided $2-3$ monitors for home PACS workstations, usually with one or two medical grade monitors and an additional monitor for other applications, including reporting software. Most (57.1\%, 24/42) workstations were fully integrated with the electronic medical record.

In $95.8 \%$ (46/48) of departments, home workstations were hosted on a hospital virtual private network (VPN). While most did not have a set departmental minimum Internet connection speed, 100 Mbps was most commonly cited among those that did (15/44, 34.1\%).

Of departments with no established rules for the geographical location of where radiologists can practice from home, $36.2 \%$ (17/47) allowed off-site work from any location, including out of state. An additional 25.5\% (12/47) of programs allowed out-of-state practice, but required departmental approval. While 8.5\% (4/47) required radiologists to be in-state, they did allow radiologists to practice from any in-state location. A minority of $29.8 \%$ (14/47) of the programs required radiologists to read from their primary residency within the same metropolitan region as the hospital.

Departmental respondents were queried regarding methods of communication; some departments provided more than one method (and more than one survey response). Most $(89.4 \%, 42 / 47)$ programs used the individual radiologists' personal cell phones or landlines for communicating with coworkers, technologists, nursing staff and other providers. However, a distinct minority mimicked internal phone communicating, creating abbreviated number codes analogous to working in the hospital in $21.3 \%$ (10/47) of departments. Least frequently, $10.6 \%(5 / 47)$ departments provided institutionally issued mobile devices.

The majority of departments (95.1\% [39/41 respondents]) used third-party applications for communication and image sharing, including Zoom (Zoom Video Communications, San Jose, CA), Microsoft Teams (Microsoft, Redmond, WA) and Webex (Cisco Systems, Milpitas, CA). Only 41.7\% (20/48) of departments provided radiologists with a video camera with the workstation to use at home. During virtual group meetings, 57.4\% (27/47) of programs indicated that fewer than half of faculty are visible on video, though for more than a third of programs $(36.2 \%, 17 / 47)$, more than $75 \%$ of faculty use video during conferences.

In $83.3 \%$ (40/48) of departments, their home PACS workstations offered identical applications in terms of thirdparty and post-processing applications as compared to in the hospital or clinic. Respondents were also asked whether additional software programs should be added to future 
configurations for home PACS workstations, beyond the standard in-hospital applications. Image capture software, reference management software such as EndNote (Clarivate Analytics, Philadelphia, PA) for research purposes, Adobe Photoshop (Adobe, Inc., San Jose, CA), MATLAB (Mathworks, Natick, MA) and other statistical analysis software were all mentioned as possible additions to home workstation setups (Online Supplementary Material 2).

For the majority of departments $(83.3 \%, 40 / 48)$, trainees are not provided home PACS workstations, though $12.5 \%$ (6/48) provide home PACS workstations to fellows, and $4.2 \%(2 / 48)$ to both residents and fellows. For the eight departments providing home workstations to trainees, five provided a completely different configuration compared to what faculty receive, with the remaining three providing the same configuration.

\section{Discussion}

\section{Overview}

Work-from-home trends in pediatric radiology rapidly evolved due to the COVID-19 pandemic. From separate SPR and SCORCH surveys of radiologists and department chairs, several important conclusions may be drawn. First, pediatric radiologists who can work from home are predominantly satisfied with their ability to work from home. Second, radiologists reported numerous improvements in working from home compared to in the hospital, particularly in terms of clinical performance and research productivity. However, radiologists perceived that their ability to teach trainees and their emotional engagement with their institution declined while working from home. Additionally, there was general agreement between individual radiologists and department chairs in the expectations of their ability to work from home after the pandemic was controlled, though there was a trend toward predicting fewer at-home assignments by department chairs compared to individual radiologists.

Individual radiologists' high reported satisfaction with working from home in our study merits consideration, particularly as rates of burnout have been shown to be high among pediatric radiologists, with work-life imbalance and call burden ranking as important stressors leading to burnout and emotional exhaustion [10-12]. It is possible that increased access to home PACS workstations during the pandemic allowed radiologists to improve work-life balance, making work from home an attractive option.

However, there were interesting differences in responses from those with and without actual experience using home PACS workstations. Specifically, relative scores related to the experience of working at home were uniformly higher for those who actually worked at home than the anticipated experience by those who did not have home PACS. While there were areas that scored as inferior when working from home compared to in the hospital, the negative impact experienced by those who worked from home was less than predicted by those who had not experienced working from home. This suggests that for those groups considering use of home PACS, the experience may be more positive than anticipated.

Despite satisfaction with home workstations, respondents reported that it was more challenging to engage with trainees remotely as compared to when in the reading room together. Our findings are consistent with previous work noting the negative impact of virtual and remote arrangements on trainees' experiences, and the disruption that residents and fellows in both radiology and other specialties have faced in their training throughout the COVID-19 pandemic $[13,14]$. This indicates special consideration must be paid to educational considerations in those groups that teach trainees and also work from home.

Also of note, while individual radiologists were generally positive about working from home, only a minority of free-text comments provided by departmental chairs were positive, suggesting a general negative outlook of the chairs toward performing clinical work from home in the long term. These free-text comments included apprehension that working from home deleteriously impacts the educational mission, the hospital-wide perception of the radiology department, and financial support from the institution. Historically, these concerns have been primarily discussed in the literature mostly in terms of contract work with an external teleradiology service provider, in particular reinforcing the notion that radiologists may be a commodity that are easily replaceable [15-19]. However, with the pandemic and the rapid change to working partially off-site for many practices (as opposed to contracting with external teleradiology providers), further discussion has included implications of hybrid practices that will undoubtedly influence the use of off-site clinical rotations in pediatric radiology practices in the future [20-23]. Notably, the department chairs' collective concerns are largely in contrast to primarily positive effects of working at home reported by surveyed pediatric radiologists, including shorter commutes, a perceived improvement in burnout, and increased flexibility for child and elder care. Consequently, while the balance of power between department leadership relative predisposition toward working at home versus individual radiologists' desires to work from home is speculatively thought more in favor of department leadership, given the widespread use of working 
from home across departments, it is considered probable that the end result will be a compromise.

\section{Considerations moving forward}

Notably, the survey indicates there was a marked change in practice in a short span of time, suggesting acceptance of a new normal in the delivery of diagnostic imaging interpretation from off-site, even if in a minority. Furthermore, nearly all responding pediatric radiology departments in the United States provide home PACS in some capacity to faculty to enable working from home. Future discussion may revolve less on whether at-home rotations will be permissible, and more on mitigating the potential negatives on trainee education, inequitable access to remote rotations for radiologists performing a higher percentage of procedural or hands-on rotations (e.g., interventional radiology, fluoroscopy, ultrasound), and impact on leadership and patient care responsibilities while maximizing benefits to the faculty to support retention and recruitment.

Both surveys support a hybrid model to ensure that pediatric radiologists get the flexibility and work-life balance benefits of home workstations, while mitigating concerns related to teaching and loss of connection with the institution. This approach would also enable sufficient on-site presence of radiologists to support leadership functions, as well as ensure visibility to other members of the radiology team. Some free-text responses in the SCORCH leadership survey suggested some ideas positively using the hybrid model. Specifically, ensuring that there is continued easy access to in-person radiologists for those consulting physicians for whom in-person interactions are preferred, but also continuing virtual rounds for those physician groups who find it more useful. Additionally, to address teaching, providing a dedicated "always on" virtual platform for use by the attending and trainee the entire day may improve collaboration and facilitate closer engagement.

The continued on-site presence also helps mitigate concerns regarding commoditization of radiology services [15-18]. Expanded coverage beyond the basic 8 a.m.-5 p.m. could also be facilitated, promoting equity by helping those with greater elder or child care responsibilities. Finally, hybrid models of staffing that allow for at-home rotations might allow senior radiologists to continue working rather than retire, helping to address the declining numbers of radiologists entering pediatric radiology [24, 25].

If at-home work is to continue, technical improvements such as embedding audiovisual connectivity programs directly in PACS to enable "one-click" communication, increased use of video cameras as part of both at-home and in-hospital PACS, improved screen-sharing capabilities beyond a single monitor, extension and standardization of audiovisual programs beyond the radiology department to include all referring providers within the institution, and increased informatics support for home PACS will be needed. Programs will also need to ensure they focus on staff wellness as remote workers may be more likely to feel disengaged from colleagues. Furthermore, while it may seem counterintuitive, those with home PACS reported there was not an increased need for wellness programs when working in-hospital compared to from home. These results may be skewed by the bias or shifting to working from home during the pandemic, with substantial additional stressors. Strategies employed at pediatric radiology departments to improve inclusion could include virtual mindfulness sessions and yoga exercises, virtual coffee breaks or happy hours to improve engagement $[23,26]$. However, only about a third of respondents found these strategies helpful, suggesting new strategies may be needed.

Additionally, perceptions of inequity also need to be addressed, as some subspecialties such as interventional radiology are more procedure-focused, or require greater on-site participation and exposure to patients, such as fluoroscopy. This discrepancy in work demands could be a source of disharmony. If some pediatric radiologists have opportunities to work from home while others cannot, an individual's at-home rotations can be a source of improved work-life balance at the cost of another's discontent.

Finally, regarding the home PACS technical setup, almost universally, either the department or hospital paid for home PACS, and a majority of home PACS systems (83\%) were equipped with applications identical to those in the hospital. As home PACS workstation configurations continue to evolve and at-home rotations become more frequent, it is expected that they will become more standardized and feature-rich, moving more into the realm of full-service home office, rather than limited to clinical PACS applications. Suggestions for improvement from SCORCH membership include reference management software, image postprocessing, statistical analysis and screen-sharing software, as well as video cameras. Image sharing and audiovisual communication software embedded within PACS or perhaps the institutional electronic health record (as opposed to a separate third-party application) and accessible via a single click, has the potential to improve discussion, understanding of complex imaging diagnoses and relationships with remote referring providers as well as education of trainees.

\section{Limitations}

This study has limitations, including constraints inherent to surveys, including recall, temporal and generalizability biases. Specifically, the results depend on the respondents' memories, which may be inaccurate. For temporal bias, the results reflect the situation at the time of the survey and may have changed since the survey date. Furthermore, the low 
response rate, particularly from SPR members, raises concern for bias. Additionally, given the low response rate, the reported differences in subjective ratings in working from home, particularly those where ratings are close to 5 on the scale of 0 to 10 , a general improvement or degradation is generally less conclusive. However, to date, this is the largest number of responding pediatric radiologists and department chairs regarding at-home practices, and can be considered the baseline for future investigations, as needed. Lastly, since the SCORCH survey respondents were pediatric radiology department chairs, their results may not be generalizable to other radiology departments. Also, similarly, given challenges in recruiting trainees to pediatric radiology, evaluating work-at-home practices in the context of other specialties would be useful to determine if what is reported and suggested here is in line with other specialties to remain attractive to recruits. While comparison with other specialties was beyond the scope of this study, it is a potential consideration for future evaluation.

Finally, the variability among programs regarding where one could site a home PACS and work remotely merits consideration. Specifically, many programs had no established geographical rules and allowed radiologists to work out of state, while others required home PACS to be sited at the faculty's primary residence and within the same metropolitan region of the hospital. It is possible that most programs had no established rules governing location of home PACS as they had not yet encountered problems requiring formal agreements and auditing of physical location. Of note, the Centers for Medicare and Medicaid Services (CMS) has established geographic practice cost indices (GPCI) for every Medicaid payment locality, which impacts the imaging exam's relative value unit (RVU). The rules concerning site of service can be based on where the radiologist interpreted the exam and can impact how GPCIs are applied in calculating the scheduled fee payment amount, but is in part based on the frequency of off-site reads [27-29]. Consequently, should home PACS remote work remain widespread among pediatric radiology practices after the pandemic, the location of a faculty's home PACS will likely be best considered in conjunction with the institution's billing and compliance department, in addition to radiology departmental leadership.

\section{Conclusion}

A majority of pediatric radiology programs used home PACS systems during the pandemic, and radiologists were satisfied with home workstations, indicating improvements in clinical and research productivity, diagnostic accuracy, and the ability to participate in departmental meetings and events when working from home. Overall, respondents who could not work from home were significantly more pessimistic about the experience of working from home compared to the respondents who worked from home. Departmental leaders were also more likely to voice concerns regarding possible commoditization of radiology services due to the ability to work from home and were more likely to feel that work should be completed in-hospital after the pandemic relative to individual pediatric radiologists. Despite this, departmental leaders' perspective on feasibility of at-home rotations has shifted, with expectation of greater numbers of at-home rotations relative to what was permissible before the pandemic.

Supplementary Information The online version contains supplementary material available at https://doi.org/10.1007/s00247-022-05299-6.

Acknowledgements Authors Matthew Seghers and Victor Seghers contributed equally to the work on this manuscript. The authors thank the Society for Pediatric Radiology leadership, Angela Davis, Laura Fenton, MD, Thierry A.G.M. Huisman, MD, Kassa Darge, MD, and Nghia (Jack) Vo, MD, for their suggestions in survey design.

\section{Declarations}

The authors declare that they had full access to all of the data in this study and the authors take complete responsibility for the integrity of the data and the accuracy of the data analysis.

Conflicts of interest None

\section{References}

1. Bokolo AJ (2021) Exploring the adoption of telemedicine and virtual software for care of outpatients during and after COVID-19 pandemic. Ir J Med Sci 190:1-10

2. Hollander JE, Carr BG (2020) Virtually perfect? Telemedicine for COVID-19. N Engl J Med 382:1679-1681

3. Okereafor K, Adebola O, Djehaiche R (2020) Exploring the potentials of telemedicine and other non-contact electronic health technologies in controlling the spread of the novel coronavirus disease (COVID-19). Ir J Med Sci 8:1-13

4. Silva E 3rd, Breslau J, Barr RM et al (2013) ACR white paper on teleradiology practice: a report from the Task Force on Teleradiology Practice. J Am Coll Radiol 10:575-585

5. Rosenkrantz AB, Hanna TN, Steenburg SD et al (2019) The current state of teleradiology across the United States: A national survey of radiologist's habits, attitudes and perceptions on teleradiology practice. J Am Coll Radiol 16:1677-1687

6. Mossa-Basha M, Meltzer CC, Kim DC et al (2020) Radiology department preparedness for COVID-19: radiology scientific expert review panel. Radiology 296:E106-E112

7. Sammer MBK, Sher AC, Huisman TAGM, Seghers VJ (2020) Response to the COVID-19 pandemic: practical guide to rapidly deploying home workstations to guarantee radiology services during quarantine, social distancing, and stay home orders. AJR Am J Roentgenol 215:1417-1420

8. Tridandapani S, Holl G, Canon CL (2020) Rapid deployment of home PACS workstations to enable social distancing in the coronavirus disease (COVID-19) era. AJR Am J Roentgenol 215:1351-1353 
9. Rosen MP, Norbash A, Kruskal J et al (2020) Impact of coronavirus disease 2019 (COVID-19) on the practice of clinical radiology. J Am Coll Radiol 17:1096-1100

10. Ayyala RS, Ahmed FS, Ruzal-Shapiro C, Taylor GA (2019) Stressors contributing to burnout amongst pediatric radiologists: results from a survey of the Society for Pediatric Radiology. Pediatr Radiol 49:714-722

11. Chen JY, Lexa FJ (2017) Baseline survey of the neuroradiology work environment in the United States with reported trends in clinical work, nonclinical work, perceptions of trainees, and burnout metrics. AJNR Am J Neuroradiol 38:1284-1291

12. Ayyala RS, Ahmed FS, Ruzal-Shapiro C, Taylor GA (2018) Prevalence of burnout among pediatric radiologists. J Am Coll Radiol $16: 518-522$

13. Alvin MD, George E, Deng F et al (2020) The impact of COVID19 on radiology trainees. Radiology 296:246-248

14. Coleman JR, Abdelsattar JM, Glocker RJ (2021) RAS-ACS COVID-19 Task Force. COVID-19 pandemic and the lived experience of surgical residents, fellows, and early-career surgeons in the American College of Surgeons. J Am Coll Surg 232:119-135

15. Boland GWL (2009) The impact of teleradiology in the United States over the last decade: driving consolidation and commoditization of radiologists and radiology services. Clin Radiol 64:457-460

16. Glazer GM, Ruiz-Wibbelsmann JA (2011) The invisible radiologist. Radiology 258:18-22

17. Forman HP, Larson DB, Kaye AD et al (2011) Masters of radiology panel discussion: the commoditization of radiology. AJR Am J Roentgenol 196:842-847

18. Gunderman R, Dodson S (2015) Is it time for radiology to embrace commoditization? J Am Coll Radiol 13:754-755

19. Krestin GP (2010) Commoditization in radiology: Threat or opportunity? Radiology 256:388-342

20. Joshi A, Garver KA, Balasubramanian S et al (2020) Childcare for radiology workers during the COVID-19 pandemic: no small matter. J Am Coll Radiol 17:1532-1534

21. Ayyala RS, Baird G, Bloom DA et al (2021) Evaluation of stress and anxiety caused by the coronavirus disease 2019 (COVID-19) pandemic in pediatric radiology. Pediatr Radiol 51:1589-1596
22. Esfahani SA, Lee A, Hu J-Y et al (2020) Challenges faced by women in radiology during the pandemic - a summary of the AAWR Women's caucus at the ACR 2020 annual meeting. Clin Imaging 68:291-294

23. Planz VB, Spalluto LB, Savoie B et al (2020) Together/apart during coronavirus disease 2019 (COVID-19): inclusion in the time of social distancing. J Am Coll Radiol 17:915-917

24. Pfeifer CM (2018) Declining interest in pediatric radiology prompts a call to action. J Am Coll Radiol 15:490-492

25. Pfeifer CM (2020) The impact of decreased Interest in pediatric radiology. J Am Coll Radiol 17:72-74

26. Moadel RM, Zamora E, Burns JG et al (2020) Remaining academically connected while socially distant: leveraging technology to support dispersed radiology and nuclear medicine training programs in the era of COVID-19. Acad Radiol 27:898-899

27. Centers for Medicare \& Medicaid Services (CMS) (2020) COVID-19 emergency declaration blanket waivers for health care providers. Updated 5/24/2021. https://www.cms.gov/files/ document/summary-covid-19-emergency-declaration-waivers. pdf. Accessed 21 July 2021

28. Centers for Medicare \& Medicaid Services (CMS) (2019) Medicare claims processing Manual: Chapter 13 - radiology services and other diagnostic procedures. https://www.cms.gov/Regul ations-and-Guidance/Guidance/Manuals/downloads/clm104c13. pdfCite ESM.. Accessed 21 July 2021

29. American College of Radiology (2020) CMS defines terms for telehealth use during COVID-19 crisis. https://www.acr.org/Advoc acy-and-Economics/Advocacy-News/Advocacy-News-Issues/Inthe-April-4-2020-Issue/CMS-Defines-Terms-for-Telehealth-UseDuring-COVID-19-Crisis/. Accessed 21 July 2021

Publisher's Note Springer Nature remains neutral with regard to jurisdictional claims in published maps and institutional affiliations.

\section{Authors and Affiliations}

\section{Matthew C. Seghers ${ }^{1} \cdot$ Victor J. Seghers ${ }^{2,3} \cdot$ Andrew C. Sher $^{2,3} \cdot$ Siddharth P. Jadhav $^{2,3} \cdot$ Lisa J. States $^{4,5}$. Andrew T. Trout ${ }^{6,7} \cdot$ Adina L. Alazraki $^{8,9} \cdot$ Marla B. K. Sammer $^{2,3}$ (D)}

1 Dell Medical School, The University of Texas at Austin, Austin, TX, USA

2 Edward B. Singleton Department of Radiology, Texas Children's Hospital, 6701 Fannin Street, Suite 470, 77030 Houston, TX, USA

3 Department of Radiology, Baylor College of Medicine, Houston, TX, USA

4 Department of Radiology, The Children's Hospital of Philadelphia, Philadelphia, PA, USA

5 Department of Radiology, University of Pennsylvania, Philadelphia, PA, USA
6 Department of Radiology, Cincinnati Children's Hospital Medical Center, Cincinnati, OH, USA

7 Department of Radiology, University of Cincinnati College of Medicine, Cincinnati, OH, USA

8 Department of Radiology, Children's Healthcare of Atlanta, Atlanta, GA, USA

9 Department of Radiology, Emory University, Atlanta, GA, USA 